\title{
Fluvastatin Increases AQP2 Urine Excretion in a Dyslipidemic Patient with Nephrogenic Diabetes Insipidus: An In Vivo and In Vitro Study
}

Giuseppe Procino1*, Maiolo $D^{2}$, Barbieri $C^{1}$, Milano $S^{1}$, Carmosino $\mathbf{M}^{1}$, Squatrito $S^{2}$, Svelto $\mathbf{M}^{1,3}$ and $G^{1}$ ullo $D^{2}$

${ }^{1}$ Department of Biosciences, Biotechnologies and Biopharmaceutics, University of Bari, Italy

${ }^{2}$ Endocrinology Unit, Garibaldi-Nesima Hospital, University of Catania Medical School, Catania, Italy

${ }^{3}$ The National Research Council (CNR), Bari, Italy

\begin{abstract}
Objective: Among the pleiotropic effects of statins, we have previously reported that fluvastatin increases the amount of plasma membrane-expressed AQP2 in renal collecting duct cells both in vitro and in vivo, independently of vasopressin. This effect may be of potential clinical significance for the treatment of patients affected by nephrogenic diabetes insipidus forms caused by inactivating mutations of the vasopressin type 2 receptor. Here we report the effect of fluvastatin on AQP2 plasma membrane abundance on an adult male XNDI patient treated with statins.

Methods: An adult male NDI patient, carrying an inactivating mutation of the V2R, under conventional treatment to reduce polyuria, was also treated with fluvastatin because of high levels of blood cholesterol. AQP2 plasma membrane expression in the kidney was monitored by measuring urinary excreted AQP2 (u-AQP2), before starting fluvastatin treatment and during a three months follow-up period. The effect of fluvastatin was also tested in vitro in mouse kidney cortical collecting duct MCD4 cells.

Results: U-AQP2 increased in a time- and dose-dependent manner after treatment with 40 and $80 \mathrm{mg} /$ day of fluvastatin for 90 days. However, at this drug dosage, increased UAQP2 was not accompanied by reduction of diuresis and increase of urine osmolality The effect of fluvastatin on AQP2 excretion was confirmed in vitro in cultured renal cells.

Conclusions: We first demonstrate that the use of fluvastatin increased AQP2 plasma membrane expression in an ND dyslipidemic patient. This observation was confirmed by in vitro studies using mouse cultured renal cells treated with fluvastatin. Although a clinical relevant effect of fluvastatin on total diuresis and urine osmolality was not observed at the used dosages, these results suggest further investigation on the possible role of HMG CoA reductase inhibitors to improve the efficacy of the current ND therapy.
\end{abstract}

\section{Introduction}

The water-conserving effect of the antidiuretic hormone Arginine Vasopressin (AVP) is mediated by its interaction with the type 2 Vasopressin Receptor (V2R), a class of G protein-coupled receptors localized at the basolateral membrane of the principal cell of the kidney collecting duct. Binding of AVP to V2R triggers the cAMP signal cascade and promotes the exocytosis of storage vesicles containing the water channel aquaporin-2 (AQP2) to the apical membrane of the kidney collecting duct principal cells [1-4].

Congenital Nephrogenic Diabetes Insipidus (NDI) is a rare disease caused by mutations of either the V2R or AQP2 genes, characterized by decreased ability of the kidney to concentrate urine in response to AVP which results in severe polyuria and polydipsia [5-10]. Mutations in the V2R gene, mapping on the X-chromosome, are causative of the X-linked NDI (XNDI) accounting for $90 \%$ of all diagnosed congenital NDI cases $[11,12]$. Affected males are unable to concentrate urine even after administration of exogenous AVP, whereas some heterozygous females, due to skewed X-chromosome inactivation, show variable degrees of symptoms $[11,13,14]$.

Limited therapeutic options are available to treat this condition. Since exogenous dDAVP is not effective, other than ensuring adequate water intake, urine volume may be partially decreased with the use of hydrochlorothiazide diuretics that, by increasing upstream salt and water reabsorption in the proximal tubule, reduce the urine load to the distal nephron where water reabsorption via AQP2 is impaired [10]. Combination of thiazide with the cyclooxygenase inhibitor indomethacin or with amiloride may also have an effect on reducing polyuria $[6,7,10,15,16]$. The above treatment may decrease urine volumes to 4-12 liters per day, which is still a large volume to void.

Many strategies have been proposed that could bypass the defective V2R signaling and restore physiological AQP2 expression and/or trafficking $[7,10,17]$. These include activation of the intracellular cGMP pathway; activation of the intracellular cAMP pathway by stimulation of the Gas-coupled calcitonin or secretin receptors; activation of E-prostanoid receptors EP2 and EP4 [18-24].

Among the plethora of candidate molecules that could improve the effect of the current therapy for XNDI, we demonstrated that statins induce an AVP-independent accumulation of AQP2 at the plasma membrane of collecting duct cells in vitro and in vivo in both wild type and XNDI mice, greatly improving kidney concentrating ability $[23,25-$ 27].

This effect on AQP2 trafficking has not been observed in humans and, in particular, on NDI patients. Here we report, for the first time, the effect of fluvastatin on AQP2 plasma membrane abundance on an adult male XNDI patient treated with statins because of high levels of blood cholesterol. AQP2 membrane expression in the kidney was monitored by measuring urinary excretion of AQP2 (u-AQP2), before starting fluvastatin treatment and for three months during treatment. u-AQP2 increased in a time- and dose-dependent manner after treatment, suggesting that the possible use of statins to improve

*Corresponding author: Giuseppe Procino, Department of Biosciences, Biotechnologies and Biopharmaceutics, Via Amendola 165/A 70126, Bari, Italy, Tel: +39 0805443414; Fax: +39 0800000000; E-mail: giuseppe.procino@uniba.it

Received June 05, 2014; Accepted July 26, 2014; Published August 03, 2014

Citation: Procino G, Maiolo D, Barbieri C, Milano S, Carmosino M, et al. (2014) Fluvastatin Increases AQP2 Urine Excretion in a Dyslipidemic Patient with Nephrogenic Diabetes Insipidus: An In Vivo and In Vitro Study. J Diabetes Metab 5: 408 doi:10.4172/2155-6156.1000408

Copyright: $\odot 2014$ Procino G, et al. This is an open-access article distributed unde the terms of the Creative Commons Attribution License, which permits unrestricted use, distribution, and reproduction in any medium, provided the original author and source are credited. 
the efficacy of the current NDI therapy is worth further investigation. Using an in vitro model of mouse cultured renal cells; we confirmed that the increased plasma membrane expression of AQP2 induced by fluvastatin is paralleled by a concomitant increase of AQP2 excretion in the culture medium.

\section{Materials and Methods}

\section{Study protocol}

A 48 year old man with mild intellectual disability was admitted to the hospital for evaluation of polydipsia and polyuria, obesity and diabetes mellitus. His weight was $100.5 \mathrm{~kg}$, height $160 \mathrm{~cm}$, BMI 38.5 $\mathrm{kg} / \mathrm{m}^{2}$; his blood pressure was $140 / 85 \mathrm{mmHg}$. He drank approximately 5 liters of water per day; urine volume was approximately 5 liters. His general clinical condition was good.

At the age of 5 he was diagnosed with non-obstructivehydronephrosis and secondary bilateral mega ureter. Water restriction test and dDAVP test revealed the presence of AVP-resistant NDI. Genetic screening revealed an AVP receptor 2 (V2R) missense mutations at nucleotide c.926T $>C$ responsible for an amino acid substitution Leu309Pro already described and responsible for XNDI [28].

He was treated with hydrochlorothiazide and amiloride with significant diuresis contraction. The current pharmacological treatment was hydrochlorothiazide $50 \mathrm{mg} /$ die and amiloride $5 \mathrm{mg} /$ die. He was previously diagnosed with diabetes mellitus and treated with metformin $1000 \mathrm{mg}$ twice a day. Exenatide $10 \mu \mathrm{g}$ twice a day s.c. was recently added. Metabolic control was good, no chronic diabetes complications were found. HBA1c was $5.8 \%$, with no glycosuria.

Other laboratory investigations revealed: sodium $142 \mathrm{mmol} / \mathrm{L}$, chloride $101 \mathrm{mmol} / \mathrm{L}$, urine osmolality $187 \mathrm{mosm} / \mathrm{kg}$, urine S.G. 1004. Thyroid function was normal. Total cholesterol was $262 \mathrm{mg} / \mathrm{dl}$ (normal range 110-200 mg/dl), HDL cholesterol was $33 \mathrm{mg} / \mathrm{dl}(35-$ 110), triglycerides were $202 \mathrm{mg} / \mathrm{dl}$ (50-150).

Cardiovascular risk factors were estimated and the patient was considered eligible for statin therapy. Fluvastatin was chosen because of our previous report showing an effect of this drug on AQP2 plasma membrane expression in the hope that this statin could also ameliorate NDI symptoms [23,27]. Informed consent was obtained prior to entering the subject into this study. The local Ethics Committee approved this observational clinical study.

\section{Fluvastatin dosing and urine sampling}

The patient was treated with fluvastatin $40 \mathrm{mg} / \mathrm{die}$ for 30 days and then the dose was increased to $80 \mathrm{mg} /$ die for the next two months. Urine samples, (24 h collection) were collected during three nonconsecutive days before initiation of the fluvastatin treatment (T0), 15 and 30 days after administration of $40 \mathrm{mg} /$ die fluvastatin (T1 and T2) and 30 and 60 days after administration of fluvastatin $80 \mathrm{mg} / \mathrm{die}$ (T3 and T4). Diuresis, electrolytes, creatinine and osmolality were measured. Urine osmolality were measured using a vapor pressure osmometer (Vapror 5520; www. wescor.com). Aliquots of each urine sample were kept at $-80^{\circ} \mathrm{C}$ until used for determination of AQP2 excretion. u-AQP2 was analyzed at each time point and values obtained at $\mathrm{T} 0$ considered as the baseline.

\section{Purification of urine exosomes}

A urine volume corresponding to $1 \mathrm{mg}$ of urine creatinine equivalents was mixed with protease inhibitors $(1 \mathrm{mM}$ phenylmethanesulfonyl fluoride, $10 \mu \mathrm{M}$ leupeptin, $1 \mu \mathrm{g} / \mathrm{ml}$ pepstatin) and centrifuged at
$3,000 \mathrm{xg}$ at $4^{\circ} \mathrm{C}$ to remove urine sediment including whole cells, large membrane fragments, and other debris. Nanomembrane concentrators Vivaspin 2,100 kDa Molecular Weight Cut-Off (MWCO) (http://www. generon.co.uk) were used. Urine was added to the nanomembrane concentrator and centrifuged at $5,000 \mathrm{xg}$ at $4{ }^{\circ} \mathrm{C}$ until the final volume obtained was approximately $20 \mu \mathrm{l}$. The retentate was solubilized in 1 volume of pre-heated $\left(95^{\circ} \mathrm{C}\right)$ Laemmli's buffer with $400 \mathrm{mM}$ DTT and proteins separated by SDS-PAGE.

\section{Cell culture and drug treatments}

Mouse cortical collecting duct MCD4 cells, stably expressing human AQP2, were generated as described elsewhere and maintained in DMEM/F12 1:1 supplemented with 5\% cholesterol-depleted Fetal Bovine Serum (FBS) [29]. Cholesterol depletion was obtained by centrifugating the FBS at $300,000 \times \mathrm{g}$ for 24 hours at $4^{\circ} \mathrm{C}$ in a BeckmanCoulter ultracentrifuge (70Ti rotor).

Cells were grown to confluence in $150 \mathrm{~cm}^{2}$ cell culture Petri dishes or on glass coverslips and treated overnight (16 h) with $50 \mu \mathrm{M}$ indomethacin (www.sigmaaldrich.com) to reduce basal cAMP levels, alone (Ctr) or in combination with either $100 \mu \mathrm{M}$ forskolin (www. sigmaaldrich.com) to stimulate cAMP production (FK), or with $5 \mu \mathrm{M}$ fluvastatin $\mathrm{Na}$ (http://www.seqchem.com) to inhibit the mevalonate pathway (Flu).

\section{Purification of exosomes from cell culture medium}

Twenty-five $\mathrm{ml}$ of cell culture medium from each experimental condition of MCD4 cells were harvested and centrifuged at 17,000xg for $30 \mathrm{~min}$ at $4^{\circ} \mathrm{C}$ to eliminate dead cells and cellular debris. The supernatants were then ultracentrifuged at $200,000 \mathrm{xg}$ for $1 \mathrm{~h}$ at $4^{\circ} \mathrm{C}$.

The pellets were washed in PBS to eliminate contaminants and resuspended in RIPA buffer. Protein concentration was determined and $5 \mu \mathrm{g}$ of total proteins were solubilized in Laemmli's buffer with 100 mM DTT, denatured at $95^{\circ} \mathrm{C}$ and separated by standard SDS-PAGE for Western blotting analysis.

\section{Immunofluorescence}

MCD4 cells, cultured on glass coverslips, were fixed with $4 \%$ Paraformaldehyde (PFA) for $15 \mathrm{~min}$ and then permeabilized with $0.1 \%$ Nonidet P-40 (www.sigmaaldrich.com) in PBS for 5 min. Nonspecific binding sites were blocked with 1\% BSA in PBS (saturation buffer) for $30 \mathrm{~min}$ at RT. Cells were then incubated with affinity-purified antibody against AQP2 $(1: 1,000)$ for $1 \mathrm{~h}$ and with AlexaFluor ${ }^{\text {mot }} 488$-conjugated donkey anti-rabbit (Invitrogen, 1:1,000) for $1 \mathrm{~h}$. Monolayers were washed, inverted on a drop of mounting medium and analysis was performed under a confocal laser-scanning fluorescence microscope (Leica TSC-SP2; www.leica.com).

\section{Immunoblotting}

Protein samples from urine and cell culture medium, solubilized in Laemmli's buffer, were resolved on $12 \%$ SDS polyacrylamide gels and electrophoretically transferred to PVDF membranes (http://www. millipore.com) for Western blotting analysis.

After blocking with 3\% Bovine Serum Albumin (BSA) in Trisbuffered saline containing $0.05 \%$ Tween 20 (TBS-T), membranes were incubated overnight at $4^{\circ} \mathrm{C}$ with primary antibodies anti-AQP2 1:1000, and aanti-Flotillin-2 1:500 (http://www.scbt.com) [29,30]. Membranes were washed in TBS-T and incubated with HRP-conjugated secondary antibodies (http://www.scbt.com) for 1 hour at room temperature. Reactive proteins were revealed with an enhanced chemiluminescent 
detection system (SuperSignal West Femto Chemiluminescent Substrate, http://www.piercenet.com) and visualized on a ChemiDoc XRS system (http://www.bio-rad.com). The quantification of protein bands was performed by determination of the relative optical density using ImageJ software (National Institutes of Health, Bethesda, MD, USA).

For exosomes isolated from cell culture media, the relative band density of AQP2 was normalized with that of Flotilin-2 band.

\section{Statistics}

Values are represented as average \pm SEM. Students' two-tailed $t$-test analysis was conducted to evaluate differences between groups. $\mathrm{P}<0.05$ was considered statistically significant.

\section{Results}

\section{In Vivo Study-Fluvastatin effect on $\mathrm{u}$-AQP2 excretion}

As shown by the Western blotting analysis reported in Figure 1A, and the relative densitometry analysis in Figure 1B, u-AQP2 levels were comparable in the three urine samples obtained before starting fluvastatin treatment (T0). After 15 days of fluvastatin treatment at 40 $\mathrm{mg} / \mathrm{die}(\mathrm{T} 1), \mathrm{u}-\mathrm{AQP} 2$ excretion was still comparable to the baseline (T0).

Interestingly, after 30 day of fluvastatin $40 \mathrm{mg} /$ die treatment (T2) $\mathrm{u}-\mathrm{AQP} 2$ increased two folds compared to the control baseline. Fluvastatin treatment at $80 \mathrm{mg} /$ die for the next 30 days (T3) led to a further dramatic dose-dependent increase of u-AQP2 excretion. At this time point AQP2 levels were nearly fourfold increase compared to baseline and remained constantly high after the last 30 days of therapy with fluvastatin $80 \mathrm{mg} /$ die (T4). However, during the entire duration of the study the increase of AQP2 excretion was not accompanied by a parallel reduction of diuresis and/or increase in urine osmolality (Figure 1C and D, respectively).

\section{In Vitro Study-Fluvastatin Effect on AQP2 Release in the Culture Medium}

In order to correlate the increased UAQP2 excretion with the increased apical membrane expression of AQP2 induced by fluvastatin, we performed in vitro studies in cultured renal cells. A mouse collecting duct cell line stably transfected with human AQP2, MDC4 cells, was used to further investigate the effect of fluvastatin on AQP2 exosome excretion. We previously showed that in this cell line incubation with fluvastatin nicely accumulates AQP2 at the apical plasma membrane [27,31]. Cells were either left untreated (Ctr) or treated with forskolin 100 $\mu \mathrm{M}$ (FK) or fluvastatin $5 \mu \mathrm{M}$ (Flu) for 16 hours in the culture medium, then subjected to immunofluorescence protocol to visualize AQP2 cellular distribution. In the same experimental conditions, exosomes excreted in the culture medium were isolated by ultracentrifugation as described in Material and methods.

As shown in Figure 2A, in resting cells, AQP2 was localized in scattered intracellular storage vesicles (Figure 1A, Ctr). FK stimulation redistributed AQP2 staining toward the apical plasma membrane (Figure 2A, FK) and fluvastatin treatment promoted a comparable effect (Figure 2A, Flu).

Figure $2 \mathrm{~B}$ and the relative densitometric analysis (Figure 2C) show the effect of forskolin and fluvastatin on AQP2 excretion in cell culture medium. Five $\mu \mathrm{g}$ of exosome-associated proteins from each experimental condition were separated by standard SDS-PAGE and
A

Western blotting analysis

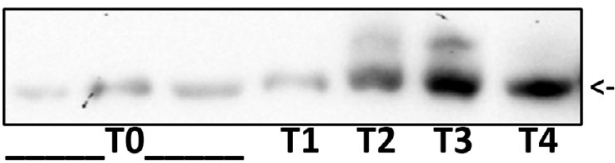

$<-A Q P 2$

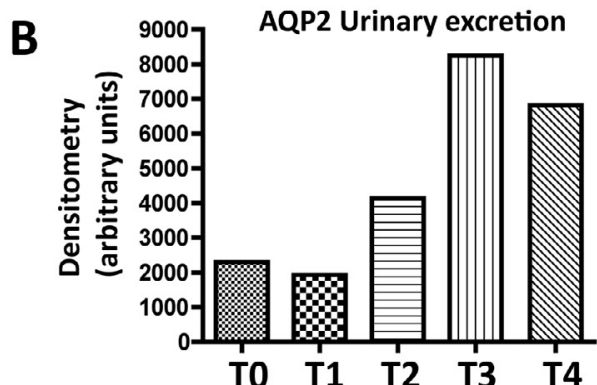

D

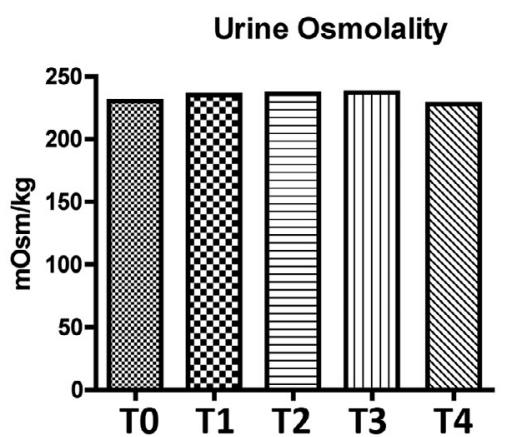

Figure 1: A) Urine samples, ( $24 \mathrm{~h}$ collection) were collected during three nonconsecutive days before initiation of the fluvastatin treatment (T0). Urine samples were also collected at days 15 (T1) and 30 (T2) after administration of $40 \mathrm{mg} /$ die fluvastatin and at days 30 (T3) and 60 (T4) after administration of fluvastatin $80 \mathrm{mg} /$ die. For each time point, a urine volume corresponding to $1 \mathrm{mg}$ of urine creatinine equivalents was processed as described in the methods section to isolate the exosome-enriched fraction. Total exosome proteins were resolved on 12\% SDS-PAGE and analyzed by Western blotting for AQP2 abundance. B) Densitometry analysis of the bands. C, D) at each time point urine output (diuresis) and urine osmolality were measured. 
A

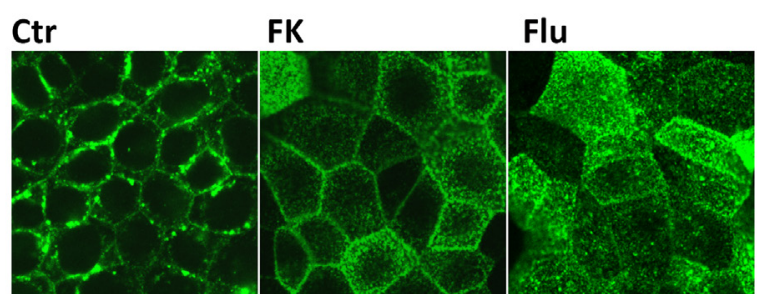

B

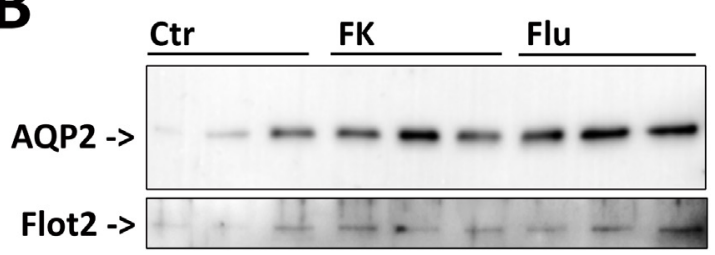

C

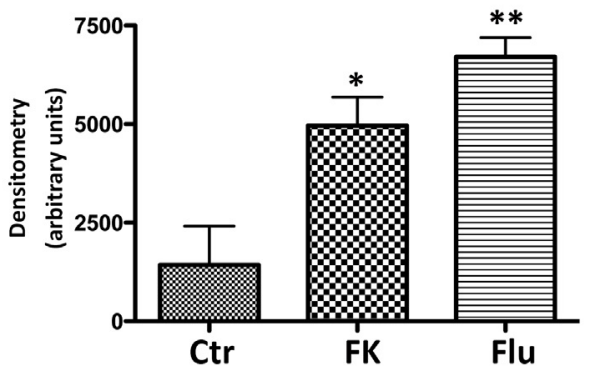

Figure 2: A) MCD4 cells were grown to confluence on glass coverslips and either left untreated (Ctr) or treated overnight $(16 \mathrm{~h}$ ) with $100 \mu \mathrm{M}$ forskolin (FK) or with $5 \mu \mathrm{M}$ fluvastatin (Flu). Cells were subjected to immunofluorescence with anti-AQP2 antibodies and analyzed in the xy plan by confocal microscopy. B) MCD4 cells were grown to confluence into $150 \mathrm{~cm}^{2}$ cell culture Petri dishes and either left untreated (Ctr) or treated overnight $(16 \mathrm{~h})$ with 100 $\mu \mathrm{M}$ forskolin (FK) or with $5 \mu \mathrm{M}$ fluvastatin (Flu). $25 \mathrm{ml}$ of culture medium from each experimental condition were recovered and exosome isolated by ultracentrifugation.

Exosomal proteins were separated by standard SDS-PAGE and analyzed by Western blotting with antibodies against AQP2 and flotillin-2 (Flot-2).

C) Densitometry of AQP2 bands was normalized for flotillin-2 abundance in each sample. Experiments were repeated three times with comparable results. ${ }^{*} p<0.05 ;{ }^{* *} p<0.001$.

analyzed by Western blotting with antibodies anti AQP2 and antiflotilin-2, an exosome marker [32,33].

As expected, FK stimulation, a classical maneuver that increases intracellular cAMP levels and promotes AQP2 exocytosis to the apical plasma membrane in renal cells, also increased AQP2 release in the culture medium via exosomes. Interestingly, fluvastatin treatment induced a dramatic increase of excreted AQP2 in the culture medium compared with unstimulated cells. This effect was even higher than that induced by forskolin. The density of each AQP2 band was normalized to that of the corresponding flotillin-2 band.

These results strongly suggest that fluvastatin increases AQP2 membrane expression in renal cells and does not interfere with AQP2 excretion outside the cell.

\section{Discussion}

We previously demonstrated that statins, in particular fluvastatin, are able to promote vasopressin-independent AQP2 plasma membrane accumulation both in vitro in renal cell culture and in vivo in wt and XNDI mice $[23,26,27]$.

In this study we provide the first experimental evidence that fluvastatin treatment is also effective in increasing AQP2 plasma membrane expression in the collecting duct renal cells in a patient affected by XNDI. This novel and interesting pleiotropic effect of statins on AQP2 trafficking may be of relevance for improving the current therapy of XNDI patients carrying inactivating mutations of the V2R receptor. We had the unique chance to observe the effect of fluvastatin treatment on AQP2 trafficking, in a hypercholesterolemic male XNDI patient subjected to fluvastatin treatment to reduce his cardiovascular risk. u-AQP2 is a well-established non-invasive urinary biomarker of collecting duct responsiveness to AVP and it is critical for the diagnosis of several disorders of renal water balance. AQP2 is excreted into the urinary space through the exosome pathway that is active at the apical membrane of most epithelial cells including the kidney tubule cells [34].

The presence of $\mathrm{u}-\mathrm{AQP} 2$ reflects the abundance of AQP2 at the apical membrane of renal cells in a number of physio-pathological states [35-38]. Accordingly, u-AQP2 increases during thirsting and after dDAVP administration, and decreases after a water load [39]. To assess whether fluvastatin was able to increase AQP2 plasma membrane expression in this patient, in which AVP signaling is abolished due to an inactivating missense mutation of the V2R, we measured $\mathrm{u}-\mathrm{AQP} 2$ before and after initiation of fluvastatin treatment [28].

Quite interestingly, the amount of $\mathrm{u}-\mathrm{AQP} 2$ was time- and dosedependently increased during fluvastatin treatment. At the end of the three months observation period, $\mathrm{u}-\mathrm{AQP} 2$ was 3- to 4 -fold increase compared to baseline. Next, we performed in vitro experiments in cultured renal cells to verify the correlation between plasma membrane expression and extracellular release of AQP2 in the presence of fluvastatin as observed in our patient. The possible interaction of statin with the both exosome pathway formation and release of plasma membrane protein outside epithelial cells is poorly understood. We took advantage of the MCD4 cells that recapitulate the major features of cortical collecting duct renal cells and are useful to study the regulated trafficking of AQP2 [29].

Overnight treatment with fluvastatin accumulates AQP2 at the apical plasma membrane of MCD4 cells to an extent comparable to that elicited by treatment with the cAMP-elevating agent forskolin, as previously reported [27]. In the same experimental conditions, we compared the amount of AQP2 released in the culture medium through the conventional exosome pathway. AQP2-containing exosomes were isolated from the culture medium by ultra-speed centrifugation and checked for the presence of the exosome marker flotillin-2 $[39,40]$. In cultured renal cells, fluvastatin-induced AQP2 accumulation at the apical plasma membrane is accompanied by a dramatic AQP2 release in the culture medium (Figure 2). This data strongly supports the conclusion that increased $\mathrm{UAQP} 2$ is a consequence of increased AQP2 plasma membrane abundance in our patient.

This is the first report showing the effect of statins treatment on AQP2 trafficking in an X-linked NDI patient. In those patients, due to inactivation of the AVP signaling, AQP2 transcription levels are very low and the amount of the channel at the plasma membrane is barely detectable $[41,42]$. The observation that fluvastatin robustly increase plasma membrane expression of AQP2, as indirectly showed by AQP2 urinary excretion, represents the first proof-of-principle in humans that statins might deserve further investigation as potential tools to improve 
Citation: Procino G, Maiolo D, Barbieri C, Milano S, Carmosino M, et al. (2014) Fluvastatin Increases AQP2 Urine Excretion in a Dyslipidemic Patient with Nephrogenic Diabetes Insipidus: An In Vivo and In Vitro Study. J Diabetes Metab 5: 408 doi:10.4172/2155-6156.1000408

Page 5 of 6

the current pharmacological treatment of XNDI. In fact, none of the drug prescribed for handling XNDI, although effective in reducing the polyuria, are targeted to reestablish AQP2 plasma membrane expression.

However, in our patient, despite the encouraging results on AQP2 membrane expression, neither urine output nor urine osmolality ameliorated, even at late point during the observation period. The lack of effect of fluvastatin on XNDI clinical signs might indicate that the pharmacological doses given in this study might be insufficient to elicit the maximal effect on AQP2 trafficking. Higher statin doses might be required to achieve pleiotropic effects beyond cholesterol lowering, especially in the kidney. To this respect, it is worth mentioning that all the statins currently available on the marked have been selected for their specific liver targeting, to maximize their cholesterol-lowering potential and reduce extra hepatic tissues effects. Different molecules of the same family, with a preferential kidney targeting, might prove more effective in promoting AQP2 trafficking.

All statins block the conversion of 3-hydroxy-3-methyl-glutarylCoA (HMG-CoA) to mevalonic acid with consequent attenuation of the biosynthesis of both cholesterol and isoprenoid intermediates of the mevalonate pathway, including farnesyl pyrophosphate and geranylgeranyl pyrophosphate $[43,44]$. Isoprenoids are important substrates for the post-translational modification (prenylation) of many signaling proteins including small GTP-binding proteins.

It has been proposed that one of the pleiotropic effect of statins, responsible for AQP2 plasma membrane accumulation in renal cells, is the inhibition of prenylation, and hence membrane anchoring, of key molecular switches regulating AQP2 trafficking in renal cells. In particular, reduced prenylation of RhoA, regulating the dynamics of cortical actin cytoskeleton polymerization and AQP2 exocytosis, might explain AQP2 accumulation at the apical plasma membrane $[27,45,46]$. In analogy, reduced prenylation of Rab5, facilitating AQP2 endocytosis at the apical plasma membrane, might stabilize AQP2 at the plasma membrane $[27,47]$.

In this scenario, specific inhibitors, blocking the mevalonate pathway downstream the first step inhibited by statins, might selectively inhibit the production of Isoprenoids without interfering with cholesterol biosynthesis. To this respect, we previously demonstrated that an inhibitor of the isoprenylation process, perillyl alcohol, mimics the effect of statins in vitro [27].

Overall, this proof-of-concept study demonstrates that fluvastatin is effective in increasing AQP2 membrane availability in the kidney of an XNDI patient. In principle, new classes of statins or higher doses of the commercially available, might achieve beneficial effects further reducing the polyuria and other clinical signs of XNDI. In conclusion, the observations described here support the hypothesis that statins or other downstream inhibitors of the mevalonate pathway might prove useful, in the future, for improving the current pharmacological treatment of XNDI.

\section{Acknowledgments}

This work was supported by fundings from the Italian Ministero della Salute/ Italian Drug Agency (project \# MRAR08P011) and by the Fondazione Telethon (project \# GGP12040) to M.S. The authors thank the following persons at the Endocrine Unit of Garibaldi-Hospital in Catania: Dr. G. Parrinello for technical assistance, Dr. V. Muscia for his help and Professor R. Vigneri for his advices on the study protocol.

\section{References}

1. Knepper MA, Inoue $T$ (1997) Regulation of aquaporin-2 water channel trafficking by vasopressin. Curr Opin Cell Biol 9: 560-564

2. Nedvetsky PI, Tamma G, Beulshausen S, Valenti G, Rosenthal W, et al. (2009) Regulation of aquaporin-2 trafficking. Handb Exp Pharmacol : 133-157.

3. Tamma G, Procino G, Svelto M, Valenti G (2012) Cell culture models and animal models for studying the patho-physiological role of renal aquaporins. Cell Mol Life Sci 69: 1931-1946.

4. Valenti G, Procino G, Tamma G, Carmosino M, Svelto M (2005) Minireview: aquaporin 2 trafficking. Endocrinology 146: 5063-5070.

5. Boone M, Deen PM (2009) Congenital nephrogenic diabetes insipidus: what can we learn from mouse models? Exp Physiol 94: 186-190.

6. Knoers N, Monnens LA (1992) Nephrogenic diabetes insipidus: clinica symptoms, pathogenesis, genetics and treatment. Pediatr Nephrol 6: 476-482.

7. Moeller HB, Rittig S, Fenton RA (2013) Nephrogenic diabetes insipidus: essential insights into the molecular background and potential therapies for treatment. Endocr Rev 34: 278-301.

8. Oksche A, Rosenthal W (1998) The molecular basis of nephrogenic diabetes insipidus. J Mol Med (Berl) 76: 326-337.

9. Robben JH, Knoers NV, Deen PM (2006) Cell biological aspects of the vasopressin type-2 receptor and aquaporin 2 water channel in nephrogenic diabetes insipidus. Am J Physiol Renal Physiol 291: F257-270.

10. Wesche D, Deen PM, Knoers NV (2012) Congenital nephrogenic diabetes insipidus: the current state of affairs. Pediatr Nephrol 27: 2183-2204.

11. Knoers NV, Deen PM (2001) Molecular and cellular defects in nephrogenic diabetes insipidus. Pediatr Nephrol 16: 1146-1152.

12. Rosenthal W, Seibold A, Antaramian A, Gilbert S, Birnbaumer M, et al. (1994) Mutations in the vasopressin V2 receptor gene in families with nephrogenic diabetes insipidus and functional expression of the Q-2 mutant. Cell Mol Biol (Noisy-le-grand) 40: 429-436.

13. Arthus MF, Lonergan M, Crumley MJ, Naumova AK, Morin D, et al. (2000) Report of 33 novel AVPR2 mutations and analysis of 117 families with X-linked nephrogenic diabetes insipidus. J Am Soc Nephrol 11: 1044-1054.

14. Faerch M, Corydon TJ, Rittig S, Christensen JH, Hertz JM, et al. (2010) Skewed $\mathrm{X}$-chromosome inactivation causing diagnostic misinterpretation in congenita nephrogenic diabetes insipidus. Scand J Urol Nephrol 44: 324-330.

15. Alon U, Chan JC (1985) Hydrochlorothiazide-amiloride in the treatment of congenital nephrogenic diabetes insipidus. Am J Nephrol 5: 9-13.

16. Kirchlechner V, Koller DY, Seidl R, Waldhauser F (1999) Treatment of nephrogenic diabetes insipidus with hydrochlorothiazide and amiloride. Arch Dis Child 80: 548-552.

17. Bouley R, Hasler U, Lu HA, Nunes P, Brown D (2008) Bypassing vasopressin receptor signaling pathways in nephrogenic diabetes insipidus. Semin Nephrol 28: 266-278.

18. Bouley R, Breton S, Sun T, McLaughlin M, Nsumu NN, et al. (2000) Nitric oxide and atrial natriuretic factor stimulate cGMP-dependent membrane insertion of aquaporin 2 in renal epithelial cells. The Journal of clinical investigation 106 1115-1126.

19. Bouley R, Pastor-Soler N, Cohen O, McLaughlin M, Breton S, et al. (2005) Stimulation of AQP2 membrane insertion in renal epithelial cells in vitro and in vivo by the cGMP phosphodiesterase inhibitor sildenafil citrate (Viagra). Am J Physiol Renal Physiol 288: F1103-1112.

20. Sanches TR, Volpini RA, Massola Shimizu MH, Bragança AC, Oshiro-Monrea F, et al. (2012) Sildenafil reduces polyuria in rats with lithium-induced NDI. Am J Physiol Renal Physiol 302: F216-225.

21. Wang W, Li C, Nejsum LN, Li H, Kim SW, et al. (2006) Biphasic effects of ANP infusion in conscious, euvolumic rats: roles of AQP2 and ENaC trafficking. Am J Physiol Renal Physiol 290: F530-541.

22. Bouley R, Lu HA, Nunes P, Da Silva N, McLaughlin M, et al. (2011) Calcitonin has a vasopressin-like effect on aquaporin-2 trafficking and urinary concentration. $J$ Am Soc Nephrol 22: 59-72.

23. Procino G, Milano S, Carmosino M, Barbieri C, Nicoletti MC, et al. (2014) Combination of secretin and fluvastatin ameliorates the polyuria associated with X-linked nephrogenic diabetes insipidus in mice. Kidney Int 86: 127-138. 
Citation: Procino G, Maiolo D, Barbieri C, Milano S, Carmosino M, et al. (2014) Fluvastatin Increases AQP2 Urine Excretion in a Dyslipidemic Patient with Nephrogenic Diabetes Insipidus: An In Vivo and In Vitro Study. J Diabetes Metab 5: 408 doi:10.4172/2155-6156.1000408

Page 6 of 6

24. Li JH, Chou CL, Li B, Gavrilova O, Eisner C, et al. (2009) A selective EP4 PGE2 receptor agonist alleviates disease in a new mouse model of $X$-linked nephrogenic diabetes insipidus. J Clin Invest 119: 3115-3126.

25. Olesen ET, Rutzler MR, Moeller HB, Praetorius HA, Fenton RA (2011) Vasopressin-independent targeting of aquaporin-2 by selective E-prostanoid receptor agonists alleviates nephrogenic diabetes insipidus. Proceedings of the National Academy of Sciences of the United States of America 108: 1294912954

26. Procino G, Barbieri C, Carmosino M, Rizzo F, Valenti G, et al. (2010) Lovastatininduced cholesterol depletion affects both apical sorting and endocytosis of aquaporin-2 in renal cells. Am J Physiol Renal Physiol 298: F266-278.

27. Procino G, Barbieri C, Carmosino M, Tamma G, Milano S, et al. (2011) Fluvastatin modulates renal water reabsorption in vivo through increased AQP2 availability at the apical plasma membrane of collecting duct cells. Pflugers Archiv : European journal of physiology 462: 753-766.

28. Shoji Y, Takahashi T, Suzuki Y, Suzuki T, Komatsu K, et al. (1998) Mutational analyses of AVPR2 gene in three Japanese families with X-linked nephrogenic diabetes insipidus: two recurrent mutations, $\mathrm{R} 137 \mathrm{H}$ and deltaV278, caused by the hypermutability at CpG dinucleotides. Hum Mutat Suppl 1: S278-283.

29. Iolascon A, Aglio V, Tamma G, D'Apolito M, Addabbo F, et al. (2007) Characterization of two novel missense mutations in the AQP2 gene causing nephrogenic diabetes insipidus. Nephron Physiol 105: p33-41.

30. Tamma G, Procino G, Strafino A, Bononi E, Meyer G, et al. (2007) Hypotonicity induces aquaporin-2 internalization and cytosol-to-membrane translocation of ICln in renal cells. Endocrinology 148: 1118-1130.

31. Procino G, Barbieri C, Tamma G, De Benedictis L, Pessin JE, et al. (2008) AQP2 exocytosis in the renal collecting duct -- involvement of SNARE isoforms and the regulatory role of Munc18b. J Cell Sci 121: 2097-2106.

32. Fitzner D, Schnaars M, van Rossum D, Krishnamoorthy G, Dibaj P, et al. (2011) Selective transfer of exosomes from oligodendrocytes to microglia by macropinocytosis. J Cell Sci 124: 447-458.

33. Street JM, Birkhoff W, Menzies RI, Webb DJ, Bailey MA, et al. (2011) Exosomal transmission of functional aquaporin 2 in kidney cortical collecting duct cells. J Physiol 589: 6119-6127.

34. Pisitkun T, Shen RF, Knepper MA (2004) Identification and proteomic profiling of exosomes in human urine. Proc Natl Acad Sci U S A 101: 13368-13373.

35. Murer L, Addabbo F, Carmosino M, Procino G, Tamma G, et al. (2004) Selective decrease in urinary aquaporin 2 and increase in prostaglandin E2 excretion is associated with postobstructive polyuria in human congenital hydronephrosis. Journal of the American Society of Nephrology : JASN 15: 2705-2712.
36. Valenti G, Fraszl W, Addabbo F, Tamma G, Procino G, et al. (2006) Water immersion is associated with an increase in aquaporin-2 excretion in healthy volunteers. Biochim Biophys Acta 1758: 1111-1116.

37. Valenti G, Laera A, Gouraud S, Pace G, Aceto G, et al. (2002) Low-calcium diet in hypercalciuric enuretic children restores AQP2 excretion and improves clinical symptoms. Am J Physiol Renal Physiol 283: F895-903.

38. Valenti G, Laera A, Pace G, Aceto G, Lospalluti ML, et al. (2000) Urinary aquaporin 2 and calciuria correlate with the severity of enuresis in children. $J$ Am Soc Nephrol 11: 1873-1881.

39. Kanno K, Sasaki S, Hirata Y, Ishikawa S, Fushimi K, et al. (1995) Urinary excretion of aquaporin-2 in patients with diabetes insipidus. N Engl J Med 332 1540-1545.

40. Aung T, Chapuy B, Vogel D, Wenzel D, Oppermann M, et al. (2011) Exosoma evasion of humoral immunotherapy in aggressive B-cell lymphoma modulated by ATP-binding cassette transporter A3. Proc Natl Acad Sci U S A 108: 15336 15341.

41. Matsumura Y, Uchida S, Rai T, Sasaki S, Marumo F (1997) Transcriptional regulation of aquaporin-2 water channel gene by cAMP. J Am Soc Nephrol 8: 861-867.

42. Yasui M, Zelenin SM, Celsi G, Aperia A (1997) Adenylate cyclase-coupled vasopressin receptor activates AQP2 promoter via a dual effect on CRE and AP1 elements. Am J Physiol 272: F443-450.

43. Greenwood J, Steinman L, Zamvil SS (2006) Statin therapy and autoimmune disease: from protein prenylation to immunomodulation. Nat Rev Immunol 6 : 358-370.

44. Bonetti PO, Lerman LO, Napoli C, Lerman A (2003) Statin effects beyond lipid lowering--are they clinically relevant? Eur Heart J 24: 225-248.

45. Tamma G, Klussmann E, Procino G, Svelto M, Rosenthal W, et al. (2003) cAMP-induced AQP2 translocation is associated with RhoA inhibition through RhoA phosphorylation and interaction with RhoGDI. J Cell Sci 116: 1519-1525.

46. Li W, Zhang Y, Bouley R, Chen Y, Matsuzaki T, et al. (2011) Simvastatin enhances aquaporin-2 surface expression and urinary concentration in vasopressin-deficient Brattleboro rats through modulation of Rho GTPase. American journal of physiology Renal physiology 301: F309-318.

47. Bucci C, Parton RG, Mather IH, Stunnenberg H, Simons K, et al. (1992) The small GTPase rab5 functions as a regulatory factor in the early endocytic pathway. Cell 70: 715-728. 